\title{
Morphological Functions with Parallel Sets for the Pore Space of X-ray CT Images of Soil Columns
}

Abstract-During the last few decades, new imaging techniques like X-ray computed tomography have made available rich and detailed information of the spatial arrangement of soil constituents, usually referred to as soil structure. Mathematical morphology provides a plethora of mathematical techniques to analyze and parameterize the geometry of soil structure. They provide a guide to design the process from image analysis to the generation of synthetic models of soil structure in order to investigate key features of flow and transport phenomena in soil. In this work, we explore the ability of morphological functions built over Minkowski functionals with parallel sets of the pore space to characterize and quantify pore space geometry of columns of intact soil. These morphological functions seem to discriminate the effects on soil pore space geometry of contrasting management practices in a Mediterranean vineyard, and they provide the first step toward identifying the statistical significance of the observed differences.

\section{Introduction}

One of the most pervasive features of natural soils is its structure as expressed by the size, shape, and arrangement of the soil particles and voids, including both the primary particles to form compound particles (i.e. soil aggregates) and the compound particles themselves (BREWER, 1964). Soil structure plays a major role in soil functioning, including its contribution to accumulation and protection of soil organic matter, to optimization of soil water and air regimes, and to storage and availability of plant nutrients

1 Depto. Matemática Aplicada a la Ing. Agronómica, E.T.S.I. Agrónomos, Universidad Politécnica de Madrid, 28040 Madrid, Spain. E-mail: fernando.sanjose@upm.es; f.j.munoz.ortega@upm.es; j.caniego@upm.es

2 Servicio de Investigación y Desarrollo Tecnológico Agroalimentario, Instituto de Ciencias de la Vid y el Vino, CSICUniversidad de La Rioja-Gobierno de la Rioja, La Rioja, 26076 Logroño, Spain. E-mail: fernandoperegrina@hotmail.com

(Bossuyt et al., 2002; vON LüTzow et al., 2006). Performance of many of these functions strongly depends on pore space geometry. For example, it has been shown that gradients of a number of soil characteristics exist inside soil. Among them are gradients in oxygen concentrations of the soil air (SEXSTONE et al., 1985), gradients in concentrations of a variety of elements, including $\mathrm{Ca}, \mathrm{Mg}, \mathrm{K}, \mathrm{Na}, \mathrm{Mn}, \mathrm{K}, \mathrm{Al}$, and Fe (SAntos et al., 1997; JASINSKA et al., 2006), and in organic matter compositions (ELLERBROCK and GERKE, 2004; URBANEK et al., 2007). These differences in turn influence soil structure that is of particular importance for processes such as soil carbon sequestration (Six et al., 2000; Denef et al., 2001; Chenu and Plante, 2006).

In this work, we propose a quantitative description of geometrical characteristics of soil pore space as volume, surface, shape, and connectivity within the unified framework that provides mathematical morphology (SERRA, 1982). Mathematical morphology includes a plethora of mathematical techniques to analyze and parameterize the geometry of different features of soil structure. These techniques belong to well-established mathematical fields such as integral geometry (SANTALó, 1976), stochastic geometry (MATHERON, 1975), or digital topology and geometry (KLetTe and Rosenfeld 2004). They make available a sound mathematical background that guides the process from image acquisition and analysis to the generation of synthetic models of soil structure (ARNS et al., 2004) to investigate key features of flow and transport phenomena in soil (LeHMAnN, 2005; MeCKE and ARns, 2005).

$\mathrm{X}$-ray computed tomography (CT) provides a direct and non-destructive procedure to use threedimensional information to quantify geometrical features of soil pore space (Peyton et al. 1994; Perret

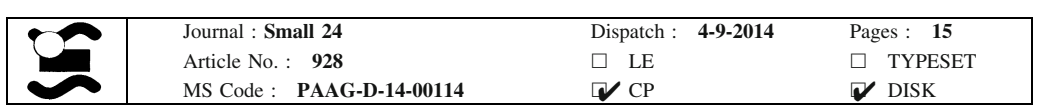


et al., 1999; Pierret et al., 2002; Mees et al., 2003; LeHMANn et al.2006; SAN José MarTínez et al., 2010; Zhou et al., 2013). During the last few decades, mathematical morphology has been successfully used to analyze different characteristics of the rich threedimensional geometrical information gained through X-ray CT (Mecke and Stoyan, 2000; Banhart, 2008). Among the tools of mathematical morphology, Minkowski functionals (ARNs et al., 2002; LEHMANN et al., 2006), which belong to the mathematical theory of integral geometry (SANTALó, 1976), are particularly worthy of consideration since they provide computationally efficient means to measure four fundamental geometrical properties of three-dimensional geometrical objects such as soil pore space. These properties are the volume, the boundary surface, the integral mean curvature, and the connectivity of the object of interest. Hadwiger's theorem (SANTALó, 1976) states that any functional that assigns a number to any three-dimensional object and meets some self-evident and natural geometrical restrictions is a linear combination of these Minkowski functionals. Then, these functionals are powerful tools to describe quantitatively 3D geometry. MeCKe (1998) and Roth et al. (2005) made use of Minkowski functions based on threshold variation of Minkowski functionals to characterize two-dimensional porous structures. San José Martínez et al. (2013) used the same methodology with the pore space of columns of intact soil. Also, two-dimensional porous structures were investigated by MECKE (2002) and Vogel et al. (2005) with Minkowski functions based on dilations and erosions. ARNs et al. (2002, 2004) considered the evolution of Minkowski functionals with dilations and erosions to characterize 3D images of Fontainebleau sandstone. Renard and Allard (San José Martínez 2013) used the Euler number as a function of erosion/dilation to explore the role of connectivity for the characterization of heterogeneous aquifers with 2D models.

In this work, we introduced two morphological transformations, namely erosion and dilations, and morphological functions built over Minkowski functionals. These morphological functions take account of the evolution of Minkowski functionals as dilations and erosions are performed on the object of interest, the pore space of soil columns imaged with X-ray CT. In this way, different geometrical objects are provided that can be seen as parallel sets of the pore space. Then, the Minkowski functionals of the new objects are computed and represented as a function of the radius of the ball of the structuring element of the corresponding dilation/erosion. We observed that morphological functions of dilation/ erosion seem to discriminate between two pore structures in a Mediterranean vineyard subjected to contrasting management practices: conventional tillage and permanent cover crop of resident vegetation.

\section{Morphology of Pore Space Volume}

Morphological analysis mimics other scientific procedures, and in some instances it can be seen as a two-step process. To illustrate this point, let us consider, for instance, the procedure to determine particle size distributions by sieving. This technique first generates a series of subsets of primary mineral particles, the oversize sets corresponding to each sieve size; then, these oversize sets are weighted. In morphological analysis, first, geometrical transformations are applied to the object of interest in an image, and then measurements are carried out. When the granulometry of an image of grains of different sizes shall be determined, successive morphological operations are performed on the image. These operations consist on the elimination of grains smaller than a certain size with a suitable morphological transformation (Fig. 1). Each one of these operations is followed by the measurement of the area for $2 \mathrm{D}$ images or the volume for 3D images, of the grains left (SERRA, 1982). Figure 1 illustrates this procedure in a CT image of a packing of sand particles. Now we are going to describe the basic morphological operations, i.e. dilations and erosions. Finally, the notions of Minkowski functionals and morphological functions will be presented.

\section{Morphological Operations}

Grains or pore space in a 3D CT image of soil will be idealized as sets of points in three-dimensional

\begin{tabular}{|l|lll|}
\hline & Journal : Small 24 & Dispatch : 4-9-2014 & Pages : 15 \\
& Article No. : 928 & $\square$ LE & $\square$ TYPESET \\
& MS Code : PAAG-D-14-00114 & $\downarrow \mathrm{CP}$ & $\checkmark$ DISK \\
\hline
\end{tabular}




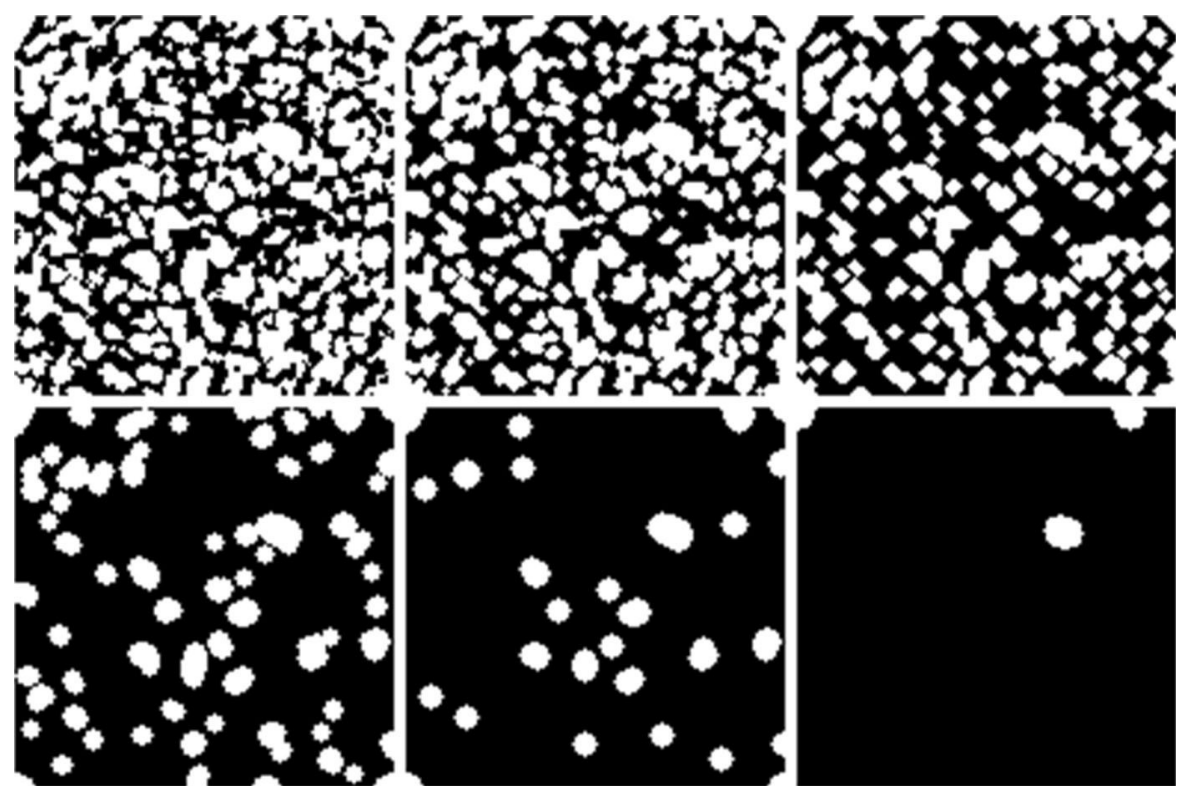

Figure 1

Granulometric analysis of a section of a CT image of $15.4 \mathrm{~mm}$ side of a packing of sand particles by successive morphological operations

space. These types of geometrical objects will be the mathematical objects of interest. In this work we will focus on soil pore space as the geometrical object of interest. Mathematically, an object is a closed and bounded set. A ball is a closed set if it contains the points of the spherical surface that defines its boundary. And it is a bounded set because it is contained in a sphere of finite radius. Dilation of an object expands it. This new object can be thought of as being the union of all balls with a given radius $r$ centered at points of the original object. If the original object is a ball of radius $r_{0}$, the dilated object by balls of radius $r$ will be a new ball of radius $r_{0}+r$.

We consider a generic object $K$ and a ball $B$ of radius 1 whose center is located at the origin of coordinates. Both $K$ and $B$ are objects, closed and bounded sets, but $K$ is the object of interest or simply an object that we scrutinize with the object $B$ that is called the structuring element. A ball of radius $r$ centered at the origin, $r B$, is obtained by multiplying the coordinates of the points of $B$ by $r$. In a ball of radius 1 , centered at point $\boldsymbol{x}, B_{x}$, is obtained adding $x$ to every point of $B$. Scalar multiplication by a positive number $r$ produces an expansion with scaling factor $r$ when $r>1$, and a contraction with scaling factor $r$ when $r<1$. Addition with a vector $\boldsymbol{x}$ produces a translation in the direction of the vector $\boldsymbol{x}$ at a distance equal to the "length" of this vector, its modulus. Then, we have the following mathematical expressions that define the sets $r B$ and $B_{x}$ (OsHer and MüCKLICH 2000):

$$
r B=\{r \boldsymbol{y}: \boldsymbol{y} \in B\} \quad \text { and } \quad B_{\boldsymbol{x}}=\{\boldsymbol{y}+\boldsymbol{x}: \boldsymbol{y} \in B\}
$$

That is to say, $r B$ is the set of points $r y$ when $y$ belongs to $B$, and $B_{x}$ is the set of points $\boldsymbol{y}+\boldsymbol{x}$ when $y$ belongs to $B$. In these expressions, $r y$ stands for the scalar multiplication of the scalar $r$ and the vector $\boldsymbol{y}$, and $\boldsymbol{y}+\boldsymbol{x}$ represents the sum of two vectors, $\boldsymbol{y}$ and $\boldsymbol{x}$. Thus, the dilation (Fig. 2) of the object $K$ by balls of radius $r$, that is the union of all balls $r B_{x}$ of radius $r$ centered at points $\boldsymbol{x}$ of $K$, will be another object $K_{r}$ defined as

$$
K_{r}=\bigcup_{x \in K} r B_{x} .
$$

The set $K_{r}$ is also called the parallel body of $K$ at a distance $r$ or $r$-parallel body to $K$. This is the set of all points within a distance smaller than $r$ from the object $K$. In this work, the structuring element will be a ball centered at the origin. Then, the dilation of an object by a ball of radius $r$ is equivalent to the $r$ -
185

186

187

188

189

190

192

193

194

195

196

197

198

199

200

202

203

204

205

206

\begin{tabular}{|l|lll|}
\hline & Journal : Small 24 & Dispatch : 4-9-2014 & Pages : 15 \\
& Article No. : 928 & $\square$ LE & $\square$ TYPESET \\
& MS Code : PAAG-D-14-00114 & $\checkmark \mathrm{CP}$ & $\checkmark$ DISK \\
\hline
\end{tabular}




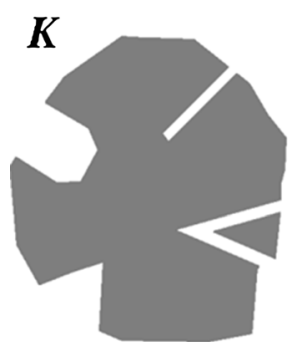

rB
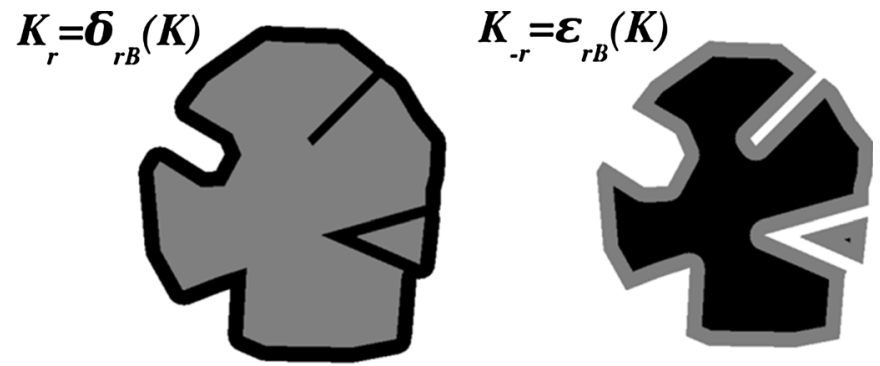

207

208

209

210

211

212

213

214

215

216

217

218

219

220

221

223

224

225

226

227

228

229

230

231 parallel body to $K$. Roughly speaking, it is like a "skin" of thickness $r$ is added to $K$.

We will analyze binary (black and white) images of soil. They contain two complementary phases: the phase of voids (pores) and the phase of soil matrix (mineral particles). As we said previously, in this study, the pore space is the object of interest and it will be white, while the mineral matrix will form the background and it will be black, as is customary in image analysis. Then, the erosion of one phase is equivalent to the dilation of the complementary phase. Erosion of the pore space is dilation of the soil matrix, and erosion of the soil matrix is dilation of pore space. For an object $K$, the erosion by a ball of radius $r$ is defined as (ARNs et al., 2002).

$$
K_{-r}=\left\{x: r B_{x} \subset K\right\}
$$

Consequently, the erosion of an object $K$ by a ball $r B$ corresponds to the set of all positions of their centers within $K$ where the structuring element $r B$ fits completely into $K$ (Fig. 2). Roughly speaking, it is like a "layer" of thickness $r$ is removed from $K$. Therefore, we may generalize the notion of $r$-parallel body so that $K_{r}$ will be a dilation for $r>0$, and erosion for $r<0$ and the original object $K$ for $r=0$ (ARNs et al., 2002).

\section{Measurements: Minkowski Functionals}

What is the area of a two-dimensional object or the volume of a three-dimensional one when the object is dilated? Let us consider a simple object like a square or a cube with edges of size $a$ and a disk or a ball of radius $r$ as a structuring element. In the plane, the area of the dilated object $K_{r}$ of a square $K$ by a disk $r B$ can easily be computed as (Fig. 3).

$$
\begin{aligned}
A\left(K_{r}\right) & =A\left(\delta_{r B}(K)\right)=a^{2}+4 a r+\pi r^{2} \\
& =A(K)+L(K) r+A(B) r^{2} .
\end{aligned}
$$

In this expression, $A$ stands for the area and $L$ stands for the length of the perimeter of the square $K$. Here, $B$ is the disk centered at the origin with radius 1. In the space, we get

$$
\begin{aligned}
V\left(K_{r}\right) & =V\left(\delta_{r B}(K)\right)=a^{3}+6 a^{2} r+3 \pi a r^{2}+\frac{4}{3} \pi r^{3} \\
& =V(K)+S(K) r+M(K) r^{2}+V(B) r^{3}
\end{aligned}
$$

Here, $V$ stands for the volume, $S$ for the area of the boundary, and $M$ for the mean breadth multiplied by $2 \pi$ (it can be shown that the mean breadth of a

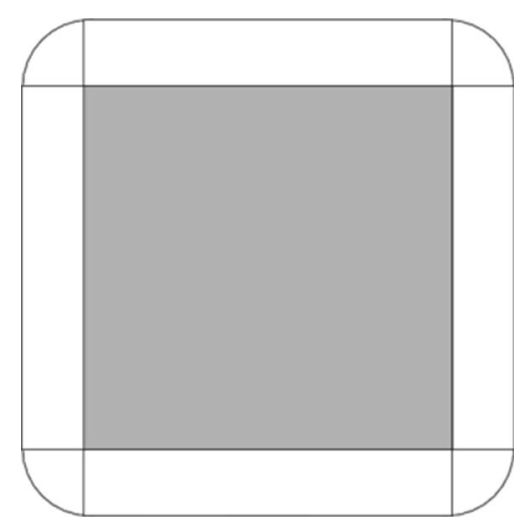

Figure 3

Dilation of a square with a disk as structuring element

\begin{tabular}{|l|lll|}
\hline & Journal : Small 24 & Dispatch : 4-9-2014 & Pages : 15 \\
& Article No. : 928 & $\square$ LE & $\square$ TYPESET \\
& MS Code : PAAG-D-14-00114 & $\downarrow \mathrm{CP}$ & $\checkmark$ DISK \\
\hline
\end{tabular}


cube of edge $a$ is $3 a / 2$ (SAntaló, 1976). Here, $B$ is the ball centered at the origin with radius 1 .

Now, let us consider a general convex object in $d$ dimensional linear space; then one has the Steiner formula (OsHER and MÜCKLICH, 2000).

$$
V\left(K_{r}\right)=\sum_{i=0}^{d}\left(\begin{array}{l}
d \\
i
\end{array}\right) W_{i}^{(d)}(K)
$$

In this expression, $W_{i}^{(d)}(K)$ are the Minkowski functionals. There are $d+1$ Minkowski functionals in dimension $d$.

Minkowski functionals are a complete set of geometrical features as established by Hadwiger's theorem (SANTALó, 1976). In simple terms, this theorem states that any functional that assigns a number to any object of interest and fulfills some very natural geometrical restrictions is a linear combination of the Minkowski functionals with numbers as scalars of this linear combination.

There are three Minkowski functionals in the plane and four in space. In the plane (the twodimensional linear space), one has

$$
\begin{gathered}
W_{0}^{(2)}(K)=A(K), W_{1}^{(2)}(K)=L(K) \quad \text { and } \\
W_{2}^{(2)}(K)=A(B) \chi(K) .
\end{gathered}
$$

In this expression, $A$ stands for the area, $L$ stands for the length of the perimeter of $K$, and $\chi(K)$ for its Euler-Poincaré characteristic. Here, $B$ is the disk centered at the origin with radius 1 . In space (the three-dimensional, linear space), one has

$$
\begin{gathered}
W_{0}^{(3)}(K)=V(K), \quad W_{1}^{(3)}(K)=(1 / 3) S(K), \\
W_{2}^{(3)}(K)=(1 / 3) M(K) \quad \text { and } \\
W_{3}^{(3)}(K)=V(B) \chi(K) .
\end{gathered}
$$

Here, $B$ is the ball centered at the origin with radius one, $V$ stands for the volume, $S$ for the area of the boundary, and $M$ for the mean breadth multiplied by $2 \pi$ (it can be shown that the mean breadth of a cube of edge $a$ is $3 a / 2$ (SAntaló, 1976). As before, $\chi(K)$ is the Euler-Poincaré characteristic of the spatial object $K$. See Appendix 2 for more details on interpretation of these functionals.

Another important feature of Minkowski functionals is that they are easy to compute (MichIELSEN 2001). For computational purposes, points of geometrical objects are considered a voxel of a digital image (i.e. the elements of regular lattice). Taking into account the $C$-additivity property (see Appendix 1) and the fact that digital images are sets of cubes (or voxels), their computation reduces to the computation of the Minkowski functionals on cubes and their intersections (vertices, edges, and faces) (Likos et al., 1995).

\section{Morphological Functions}

Mathematical morphology offers a powerful description of objects in terms of functions. This technique is similar to the process that provides particle size distributions by morphological analysis of soil images (Serra, 1982; Sollle, 2002; Vogel, 2002).

Consider a 3D binary image of soil where the void phase $\mathrm{K}$ is the object of interest. Let $K_{r}$ be, as before, the dilation of $K$ by balls of radius $r$ when $r>0$ and the erosion of $K$ by balls of radius $r$ when $r<0$. Then, consider any Minkowski functional, say $M$, and the function

$$
f(r)=M\left(K_{r}\right)
$$

This family of functions built over the Minkowski functionals provides a way to investigate the morphology of the pore space $K$ as it is dilated and eroded with balls of increasing radius $r$. Vogel $e t$ al. (2005) used this approach on 2D images to describe crack dynamics in clay soil. Rотн et al. (2005) make use of opening (i.e. erosion followed by dilation) to build Minkowski functions to quantifying permafrost patterns with aerial photographs. These functions add new information to that provided by Minkowski functionals as they yield the pore size distribution of the porous structure. ARNs et al. (2004) characterized disordered systems and matched model reconstructions to 3D images of Fontainebleau sandstone with Minkowski functions based on dilations and erosions. Vogel et al. (2010) took advantage of Minkowski functions based on openings to quantify soil structure of arable soil and of repacked sand using 3D images from X-ray tomography of samples of different sizes recorded at different resolutions.
296

297

298

299

300

301

302

303

304

305

306

307

\begin{tabular}{|l|lll|}
\hline & Journal : Small 24 & Dispatch : 4-9-2014 & Pages : 15 \\
& Article No. : 928 & $\square$ LE & $\square$ TYPESET \\
& MS Code : PAAG-D-14-00114 & $\downarrow \mathrm{CP}$ & $\checkmark$ DISK \\
\hline
\end{tabular}


MeCKe (1996) considered a different type of Minkowski function. In this case, the original 2D image is a grayscale image before segmentation. A series of binary images were obtained when the threshold varied from the minimum value of the grayscale to its maximum. Minkowski functionals were evaluated on each binarized image of the series, and four Minkowski functions were defined when the Minkowski functionals evolved as a function of threshold. Rотн et al. (2005) also made use of this type of functions to quantify permafrost patterns obtained from aerial 2D photographs.

In this work, we will investigate, in a threedimensional setting, how Minkowski functions based on parallel sets of binary 3D X-ray CT images of soil columns can be used to characterize soil pore structure of cultivated soil.

\section{Materials and Methods}

\subsection{Soil Columns: Sample Collection}

The columns were collected at the experimental farm "Finca La Grajera", a property of La Rioja region government, northern Spain, Latitude, $42^{\circ} 26^{\prime} 3418^{\prime \prime} \mathrm{N}$; longitude $2^{\circ} 30^{\prime} 5307^{\prime \prime} \mathrm{W}$, in December 2010. The field slope was about $10.2 \%$ with west-east orientation. The soil was classified as fineloamy, mixed, thermic Typic Haploxerepts according to the USDA soil classification (Soil Survey Staff, 2006), and contained $230 \mathrm{~g} \mathrm{~kg}^{-1}$ clay, $433 \mathrm{~g} \mathrm{~kg}^{-1}$ silt, $337 \mathrm{~g} \mathrm{~kg}^{-1}$ sand, $9.3 \mathrm{~g} \mathrm{~kg}^{-1}$ organic matter, and $149 \mathrm{~g} \mathrm{~kg}^{-1}$ carbonates, with $\mathrm{pH} 8.62$ and electrical conductivity $0.17 \mathrm{dS} \mathrm{m}^{-1}$ at the Ap horizon $(0-20 \mathrm{~cm})$. Climate in the area is semiarid according to the UNESCO aridity index (UNESCO, 1979), with heavy winter rains and summer drought conditions. For the period 2005-2009, the average annual precipitation was $470 \mathrm{~mm}$, average annual temperature was $13{ }^{\circ} \mathrm{C}$, and average annual potential evapotranspiration (FAO-Penman) was 1,132 mm.

In this study, we considered four columns collected between rows of the vineyard that was established in 1996 with Vitis vinifera L. "Tempranillo", grafted onto 110-R rootstock. Two types of soil cover management in between rows were undertaken:
( $T$ ) conventional tillage management between rows, which consisted of a soil tillage of 15-cm depth by cultivator once every 4-6 weeks, as required for weed control during the grapevine growth cycle; (C) permanent cover crop of resident vegetation, which was dominated by annual grass and forbs common to La Rioja vineyards (see Peregrina et al., 2010, for more details). Columns were extracted vertically by percussion drilling between rows, within PVC cylinders of $7.5 \mathrm{~cm}$ interior diameter and $30 \mathrm{~cm}$ height from the upmost part of soil profile. As a consequence, only the upper half of the column was affected by tillage that was undertaken 3 months before the collection of samples.

\subsection{Image Acquisition, Filtering, and Segmentation}

Soil columns were scanned at Fraunhofer ITWM facilities (Germany) with a PerkinElmer amorphous silicon (a-Si) detector with $2,048 \times 2,048$ pixels and a Feinfocus FXE 225.51 microfocus beam source tube. It was operated at $190 \mathrm{kV}(53 \mu \mathrm{A})$ acceleration voltage and $20 \mathrm{~W}$ target power. The tube had a tungsten target installed. In addition, a collimator to reduce stray radiation and a $200-\mu \mathrm{m}$ steel filter in front of the target was used. Only the upper half of the column was scanned to image the tilled part of the columns from tilled soil, and the region between 6.5 and $15 \mathrm{~cm}$ was selected to have a resolution of $50 \mu \mathrm{m}$. In this way, soil macro-pore structure important for intense renewal of air and serving to transport and distribute water in soil (BREWER, 1964) was imaged.

Raw data from tomography correspond to a stack of 1,706 two-dimensional, 16-bit grayscale images with a pixel size of $50 \mu \mathrm{m}$. These horizontal sections are disks of $7.5 \mathrm{~cm}$ diameter, $50 \mu \mathrm{m}$ apart from one another. Thus, the 3D image is made up of voxels of $50 \mu \mathrm{m}$. Light values of the grayscale designate voxels corresponding to low densities of the soil column, whereas high values indicate voxels of high density parts of the column. The original 2D projections were filtered by a $3 \times 3$ median filter before reconstruction in order to reduce random noise from the detector. It is a nonlinear smoothing method used to reduce isolated noise without blurring sharp edges (W and LAI, 2009).

\begin{tabular}{|l|lll|}
\hline & Journal : Small 24 & Dispatch : 4-9-2014 & Pages : 15 \\
& Article No. : 928 & $\square \mathrm{LE}$ & $\square$ TYPESET \\
& MS Code : PAAG-D-14-00114 & $\square \mathrm{CP}$ & $\checkmark$ DISK \\
\hline
\end{tabular}


The segmentation process provides a way to separate the object of interest from the background, in this case, the pore space from the soil matrix. This process produces binary images when a threshold is selected, and every voxel with a grayscale value lower than the selected threshold is considered part of the pore space and set to 1 (white), while every voxel with a grayscale value higher than the selected threshold is considered part of the soil matrix and set to 0 (black). ImageJ version $1.47 \mathrm{v}$, a public domain program developed at the National Institutes of Health, was used for image processing. We selected a global method as we focused primarily on the analysis of geometrical features evolutions. The modes method of thresholding was chosen to generate binary images (SonKA et al., 1998) for its performance (IASSANOv et al., 2009). In this procedure, the histogram is iteratively smoothed until there are only two local maxima. Then, the threshold is chosen at the midpoint between these local maxima. Figure 4 illustrates image binarization, and Fig. 5 shows the view of $3 \mathrm{D}$ reconstruction of pore space in a binary image. The plot of histograms with logarithmic scale on the vertical axis is displayed (Fig. 4) to show the two maxima. Notice the different pore structures that display a typical sample from soil under cover crop of resident vegetation and from soil under conventional tillage (Fig. 5). The homogeneity of the pore space produced by tillage is obvious ( $\mathrm{T}$ samples) as compared to the much more heterogeneous result of the cover resident vegetation crop (C samples).

\subsection{Computing Minkowski Functions for Parallel Sets}

We will consider binary images segmented with the modes method procedure. In these images, the pore space will be the object of interest while the soil matrix will be the background. Now, to study pore structure, we will investigate the evolution of Minkowski functionals as successive erosions, and dilations with balls of increasing radius are performed on the binary images (ARNs et al., 2002; VoGel et al. 2005).

We follow the procedure developed by MECKE (1996) and the code published by Michielsen (2001) to compute Minkowski functionals. For the sake of
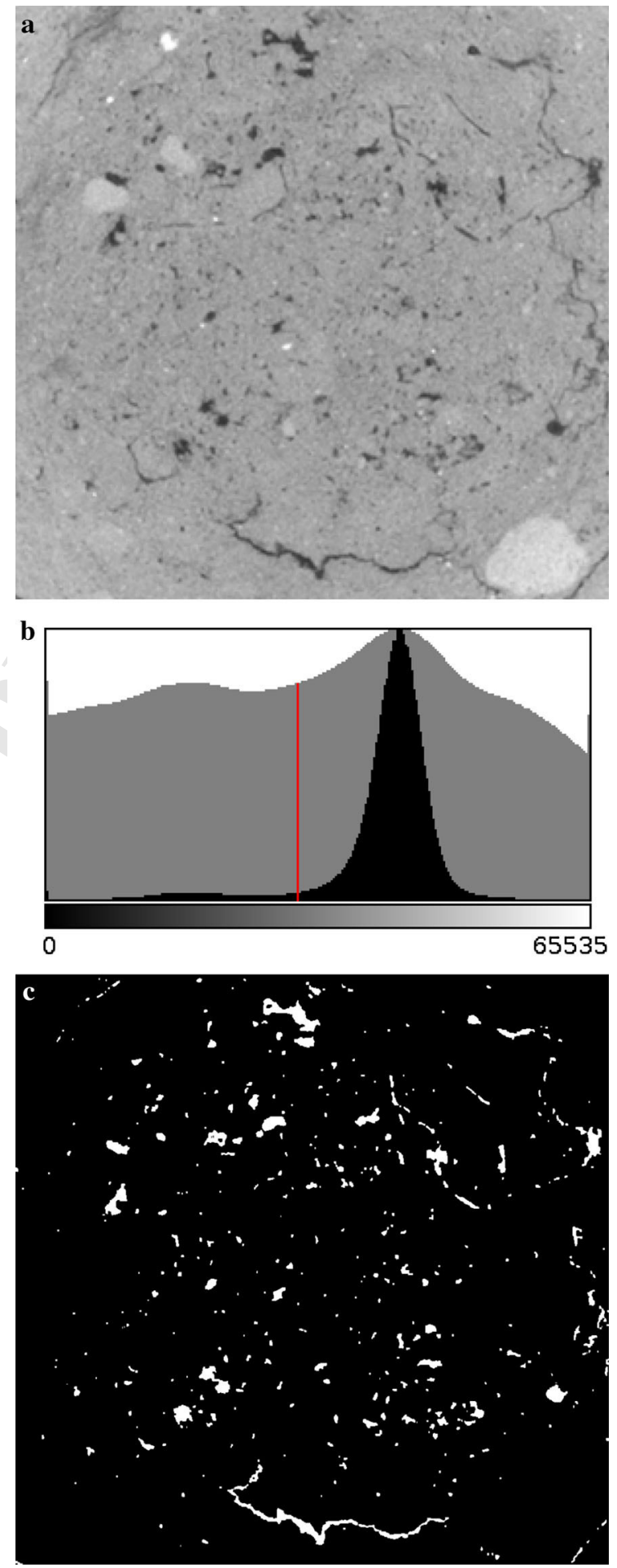

Figure 4

Segmentation process on a horizontal section of $960 \times 960$ pixels of column C1: a gray-scale image, b histogram with (black) and without (grey) logarithmic scales, and the resulting threshold marked with a vertical red line, and $\mathbf{c}$ segmented image (white voids, black solid)

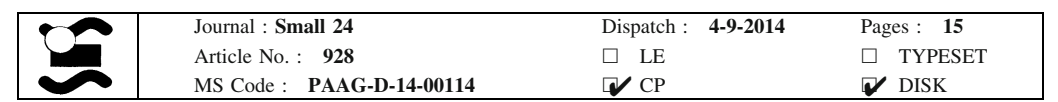




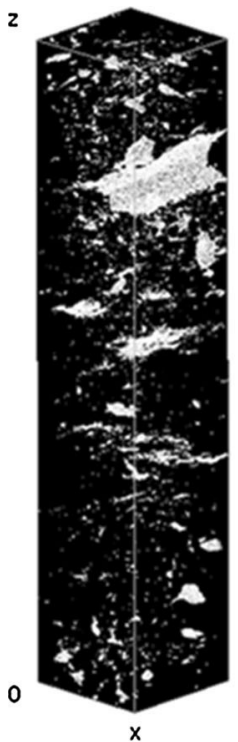

$\mathrm{T} 1$

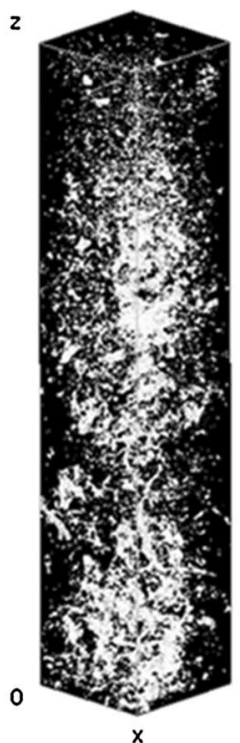

$\mathrm{T} 2$

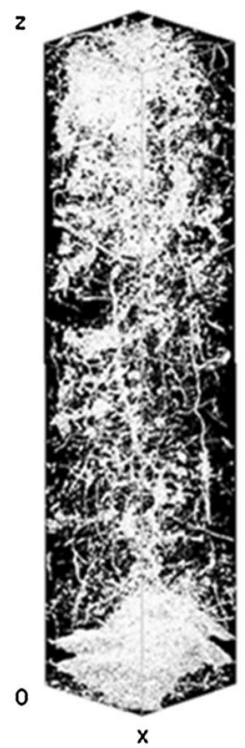

C1

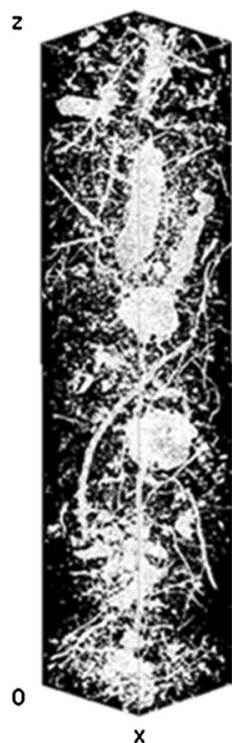

C2

Figure 5

3D reconstructions of the pore geometry (white) in each soil column in a box that is $8.5 \mathrm{~cm}$ high ( $z$ axis) and $1.7 \mathrm{~cm}$ long ( $x$ axis) and wide ( $y$ axis)

462 clarity, let us illustrate this procedure in 2D images 463 made up of pixels that geometrically are squares. The 464 object of interest, $\mathrm{K}$, is a finite union of squares 465 (compact and convex object). Each square is consid466 ered to be decomposed into the four points of its four 467 vertices, the four open segments of its four edges, and 468 the rest of the square, i.e. its interior. Then, the square 469 of each pixel is the union of nine disjoint sets: four 470 points, four open segments, and the interior of the 471 square. As a consequence, we only need to know the 472 Minkowski functional of these three types of sets (a 473 point, an open segment, and an open square), and 474 then use C-additivity extended to the union of an 475 arbitrary amount of sets. If $n_{s}$ is the number of 476 squares of the object, $n_{e}$ the number of edges, and $n_{v}$ 477 the number of vertices of the pixels of the object of 478 interest are counted once, it is easy to verify that 479 (MichiELSEN 2001).

$$
\begin{aligned}
& A(K)=n_{s}, \quad L(K)=-4 n_{s}+2 n_{e} \quad \text { and } \\
& \chi(K)=n_{s}-n_{e}+n_{v} .
\end{aligned}
$$

480 For three-dimensional objects, a similar argument 482 shows that (MichIELSEN 2001).

$$
\begin{aligned}
& V(K)=n_{c}, \quad S(K)=-6 n_{c}+2 n_{f}, \\
& \pi^{-1} M(K)=3 n_{c}-2 n_{f}+n_{e} \quad \text { and } \\
& \chi(K)=-n_{c}+n_{f}-n_{e}+n_{v}
\end{aligned}
$$

In this expression, $n_{c}$ is the number of cubes and $n_{f}$ is the number of faces of the voxels of the object $K$, counted once.

The Euler-Poincaré characteristic-Euler number, for short-describes the connectivity of an object. In order to reconcile this global topological point of view with the local counterpart that displays the computation of this number in terms of numbers of cubes, faces, edges, and vertices, it is necessary to define when voxels are connected, or equivalently, when are they neighbors. In the plane, a common choice is to consider that two black pixels are connected when they have an edge or a vertex in common. In the three-dimensional space, it is customary to consider two black voxels connected when they have a face, an edge, or a vertex in common. This implies that any voxel is connected to 26 voxels or it has 26 neighbors (MiCHIELSEN and DE RAEDT, 2001).
483

485

486

487

488

489

490

491

492

493

494

495

496

497

498

499

500

501

502

\begin{tabular}{|l|lll|}
\hline & Journal : Small 24 & Dispatch : 4-9-2014 & Pages : 15 \\
& Article No. : 928 & $\square$ LE & $\square$ TYPESET \\
& MS Code : PAAG-D-14-00114 & $\downarrow \mathrm{CP}$ & $\checkmark$ DISK \\
\hline
\end{tabular}




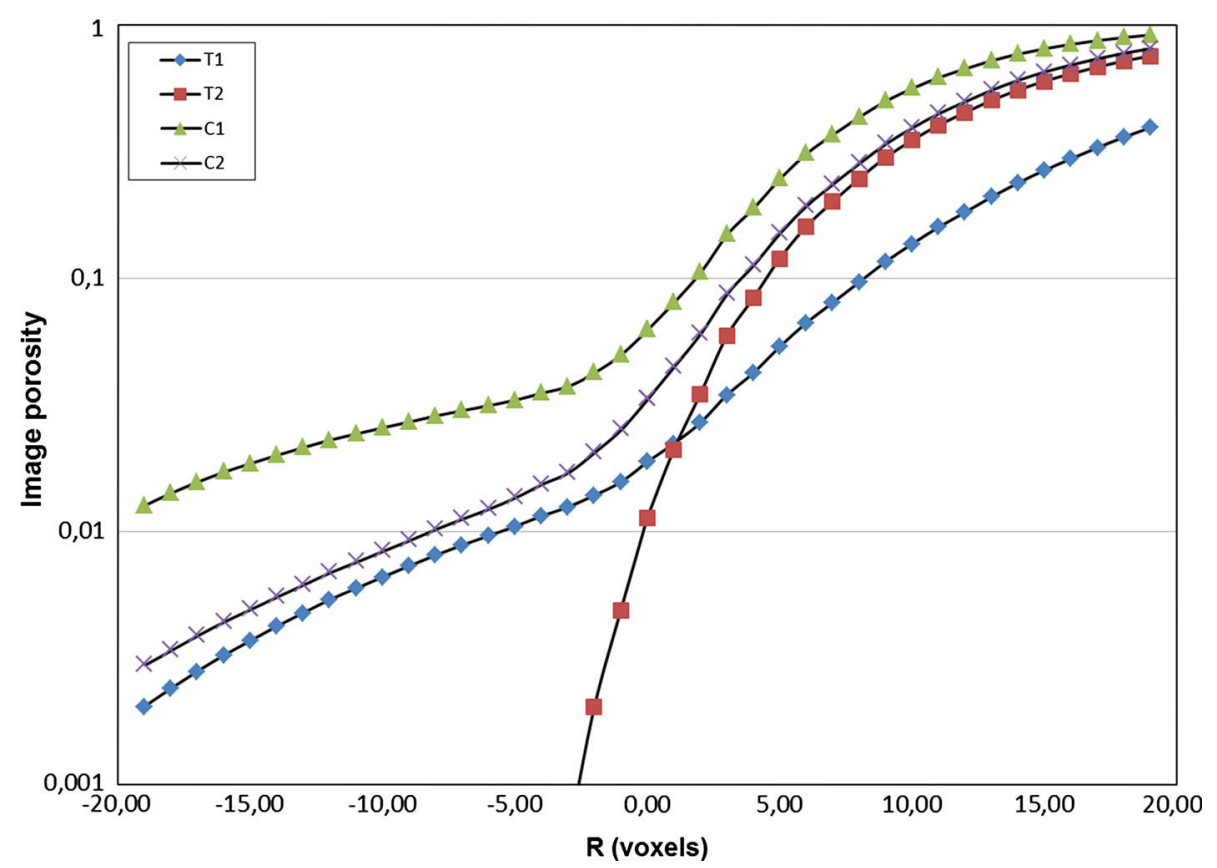

Figure 6

Image porosity as a function of diameter of erosion/dilatation

\section{Results and Discussion}

To evaluate Minkowski functionals, each column was divided into five consecutive cubes that shared a face, from top to bottom. The cubes had 340 voxels per edge and they were centered on the axes of the column in order to avoid voxels belonging to the container or voxels representing soil near the sampling tube that might have been damaged during sampling. The pore space in each cube was eroded/ dilated to yield parallel sets. Diameters of balls took 19 different values for erosions and 19 for dilation, as well; it was incremented from 0 in steps of the voxel size (i.e. $50 \mu \mathrm{m}$ ). As Minkowski functionals are additive, their values for each column were obtained by simply adding the corresponding values of the cubes of the column. We considered densities of Minkowski functionals. Thus, we had volume fraction or image porosity, specific boundary surface area, specific integral of mean curvature, and specific Euler number of the pore space.

Figures $6,7,8,9$ display the evolution of these geometrical densities as functions of erosion/dilation diameter $(R)$. As stated above, dilations of pore space produce an increase of its volume. Let us remark that this effect is more pronounced when there are tunnels of soil materials through voids because dilations reduce them, even if it also depends on the complexity of the pore-solid interface as measured by surface area and integral of mean curvature. Roughly speaking, dilations turn some voxels of the soil matrix into voxels of its pore space. Hence, this morphological operation expands the void part of the sample. Erosion produces the inverse process. Differences between soil samples under natural resident vegetation cover $(\mathrm{C})$ and samples under conventional tillage (T) are noticeable even if samples $\mathrm{T} 2$ and $\mathrm{C} 2$ have a similar evolution for dilations. Nevertheless, the evolution of image porosity (Fig. 6) and specific boundary surface (Fig. 7) with erosions diverges. This suggests that geometrical features of sample T2 are smaller than three voxels as they vanish with erosions of diameter smaller than that size. The opposite behavior is observed on sample $\mathrm{C} 1$. The erosion with the larger ball still left an important amount of porosity in this sample. Overall, samples with natural resident vegetation cover (C) store a greater amount of volume fraction and specific surface at any diameter of the balls used to erode/dilate as compared to samples from tilled soil (T). This is

\begin{tabular}{|l|lll|}
\hline & Journal : Small 24 & Dispatch : 4-9-2014 & Pages : 15 \\
& Article No. : 928 & $\square$ LE & $\square$ TYPESET \\
& MS Code : PAAG-D-14-00114 & $\downarrow \mathrm{CP}$ & $\checkmark$ DISK \\
\hline
\end{tabular}




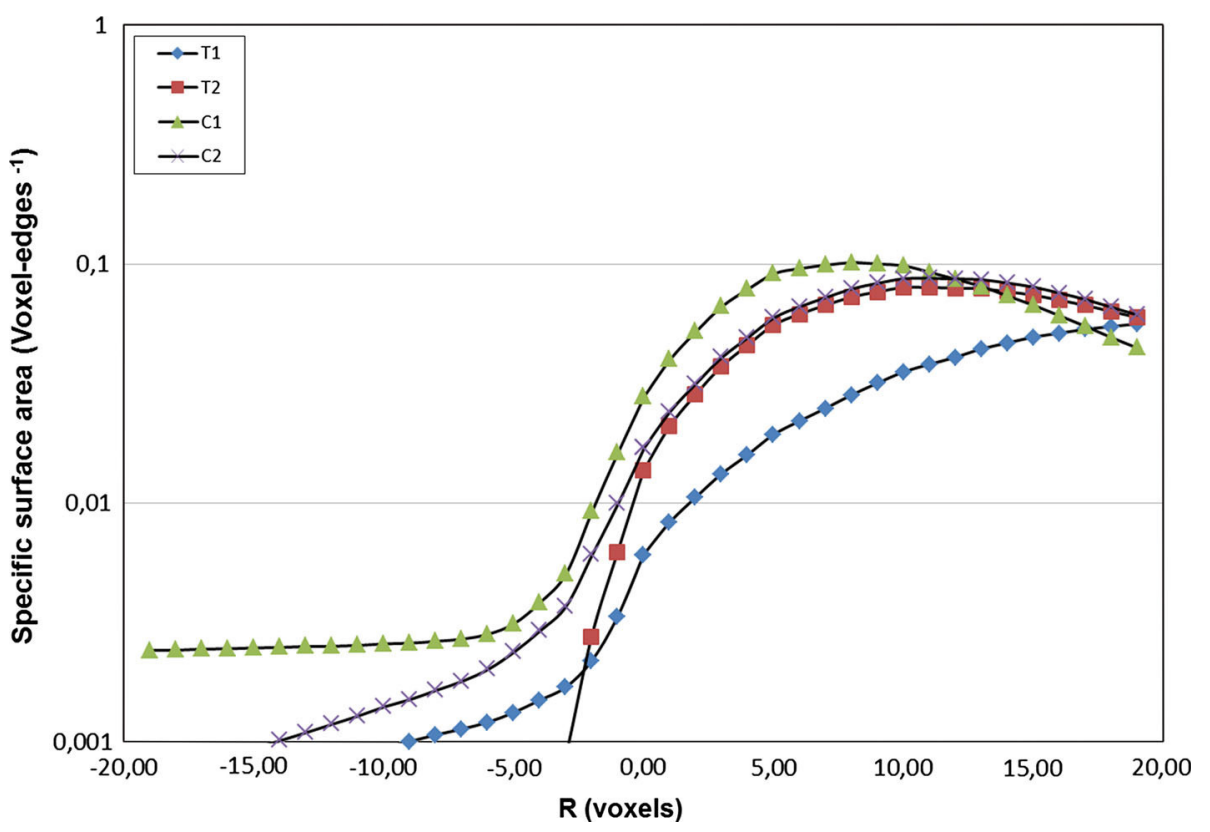

Figure 7

Specific surface area (voxel-edges ${ }^{-1}$ ) as a function of diameter of erosion/dilatation

552 consistent with results reported by PEREGRINA et al. 553 (2010).

$554 \quad$ Figures 8 and 9 depict the evolution of the specific 555 integral of mean curvature-mean curvature, for 556 short-and connectivity. Let us remember that the 557 connectivity is evaluated as the number of connected 558 components of the object of interest minus its tunnels 559 plus its cavities (see Appendix 2). Tunnels are 560 redundant loops or handles, as torus-like holes 561 through the object of interest. As we are dealing with 562 images of a natural soil, we may assume that there are 563 no soil materials completely surrounded by voids and, 564 as a consequence, the Euler number corresponds to the 565 number of connected components of the pores space 566 minus the number of tunnels of solid materials 567 through the pore space. The morphological functions 568 of the specific mean curvature (Fig. 8) and connec569 tivity (Fig. 9) seem to indicate that conventional 570 tillage and resident vegetation cover produces two 571 different pore structures; this difference is especially 572 apparent when comparing samples $\mathrm{C} 1$ and T1. Sample 573 C1 yields more specific mean curvature than sample 574 T1 when dilated with balls smaller than nine voxels. 575 In this range of diameters, mostly small voids con576 necting soil matrix should populate sample $\mathrm{C} 1$ as compared to sample T1, as is apparent from Fig. 5. 577 High Euler numbers of sample $\mathrm{C} 1$ at small diameters $\quad 578$ seem to suggest this behavior. But large diameters 579 decrease specific mean curvature and Euler number of $\quad 580$ sample $\mathrm{C} 1$, producing negative values. Nevertheless, 581 in the case of $\mathrm{T} 1$, these geometrical measurements 582 have lower growth. In the case of connectivity, it is 583 negative for the largest diameter of dilations. This 584 suggests that the pore structure of sample $\mathrm{C} 1$ contains $\quad 585$ a great amount of small features as the number of 586 small voids (i.e. connected components) exceeds the 587 number of tunnels of solid materials through them; 588 therefore, high values of the specific mean curvature $\quad 589$ from these small features of the $\mathrm{C} 1$ pore space might $\quad 590$ be explained by the regularity of the surface that 591 enclosed them, and they are also compatible with their $\quad 592$ small size. Moreover, C1 seems to display a rich 593 structure as compared to sample T1. Between diam- 594 eters 8 and 9, the graphs of both samples intersect at a 595 positive specific mean curvature, but sample $\mathrm{C} 1$ has 596 negative Euler characteristic. Therefore, it suggests 597 that geometrical features similar in size should dom- 598 inate sample T1, while the dilations of sample C1 599 show a more complex structure highly connected with $\quad 600$ tunnels through it, as it seems to indicate negative 601

\begin{tabular}{|l|lll|}
\hline & Journal : Small 24 & Dispatch : 4-9-2014 & Pages : 15 \\
& Article No. : 928 & $\square \mathrm{LE}$ & $\square$ TYPESET \\
& MS Code : PAAG-D-14-00114 & $\square \mathrm{CP}$ & $\checkmark$ DISK \\
\hline
\end{tabular}




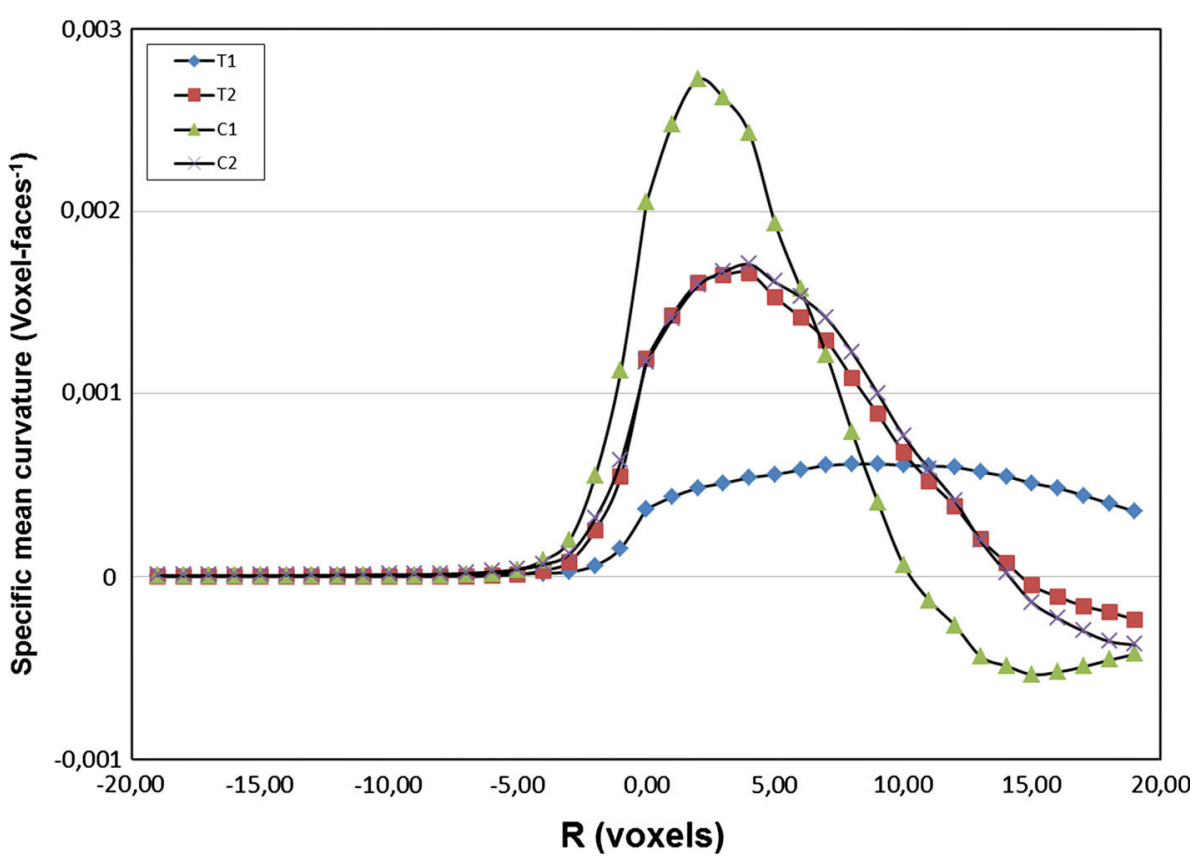

Figure 8

Specific curvature (voxel-faces ${ }^{-1}$ ) as a function of diameter of erosion/dilatation

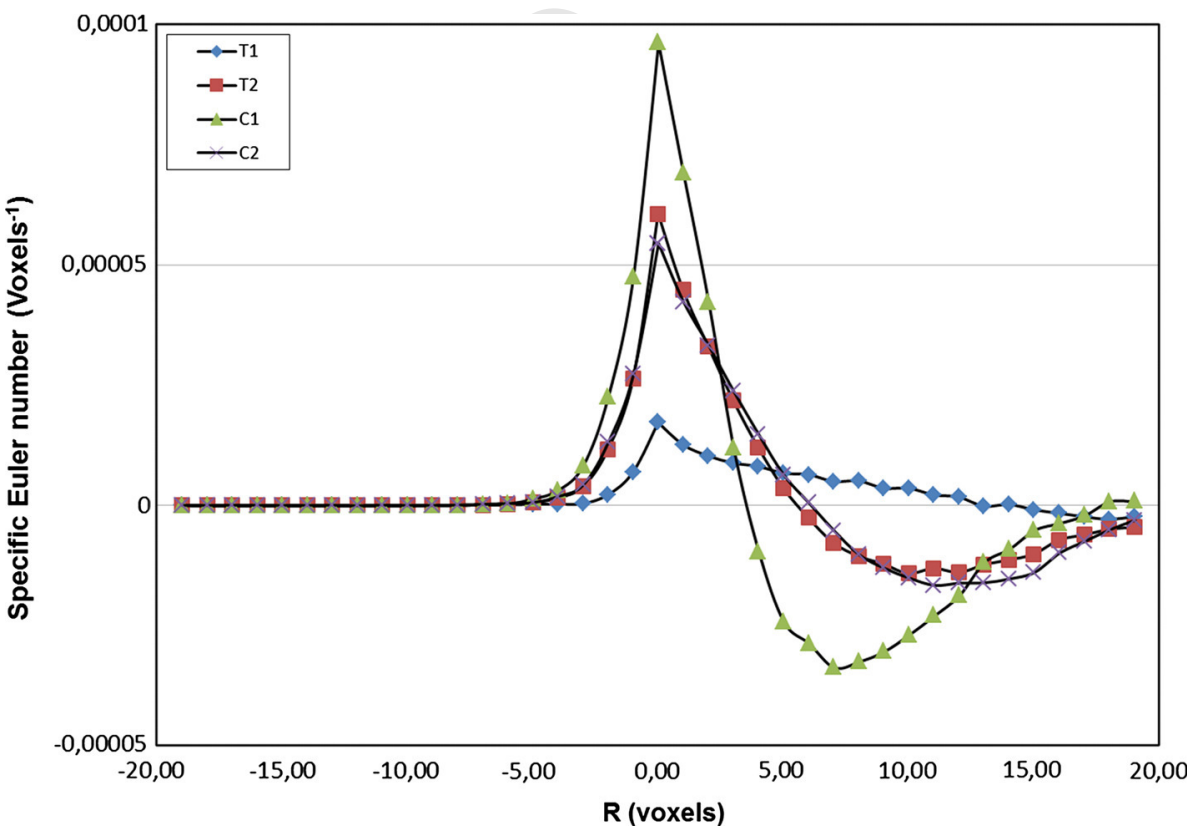

Figure 9

Specific Euler number $\left(\right.$ voxel $^{-1}$ ) as a function of diameter of erosion/dilatation

602 Euler numbers. The low variation of specific mean 603 curvature and Euler numbers of sample T1 is com-
604 patible with a pore structure made up with irregular 604 geometrical features of similar sizes that collapse as diameter of dilation increases and do not generate a complex and highly connected structure.

\begin{tabular}{|l|lll|}
\hline & Journal : Small 24 & Dispatch : 4-9-2014 & Pages : 15 \\
& Article No. : 928 & $\square$ LE & $\square$ TYPESET \\
MS Code : PAAG-D-14-00114 & $\square \mathrm{CP}$ & $\downarrow$ DISK \\
\hline
\end{tabular}


608 These results open the door to new investigations 609 to identify statistically significant differences in soil 610 structure due to contrasting management practices. It 611 was the necessary first step towards further research 612 that should include a richer sample. Then, the trends 613 that suggest this study would be the hypothesis of 614 those new investigations. Therefore, this might pro615 vide the basis for new projects that are likely to be lengthy and costly, as there is the need for a greater amount of 3D tomograms of large soil columns.

It has been reported that different land use and management practices significantly affect directions and magnitudes of the soil processes by contributing different quantities and qualities of biomass inputs, generating different levels of soil disturbance, influencing soil temperature and moisture regimes. These differences generate notable changes in soil physical and hydraulic properties, including changes in soil organic matter content, soil porosity, hydraulic conductivity, and water retention (WANG et al., 2012; Zhou et al., 2013). Our results suggest that the evolution of morphological features with dilation/erosion is a suitable indicator of soil structure for cultivated soil, and it seems to describe the influence of two different soil management practices (i.e. conventional tillage and natural cover crop) on soil structure in a Spanish Mediterranean vineyard. It is worth noting here how these results reflect the different pore structures as depicted by Fig. 5. The homogeneity of the pore space produced by tillage is obvious as compared to the heterogeneity of samples under resident vegetation cover. Similar geometrical features seem to dominate samples $\mathrm{T} 2$ and $\mathrm{C} 2$, but big structures discriminate between them and explain the behavior of the morphological functions of image porosity and specific boundary surface when sample T2 is eroded. These results are consistent with previous studies on the impact of land use on soil structure (KRAVCHENKO et al., 2011; WANG et al., 2012) when they remarked on the homogeneity of the pore structure of conventional tillage as compared with no-till.

Soil structure is regarded as one of the main providers of physical protection of soil organic matter and carbon sequestration by soils (SIX et al., 1998). One of the mechanisms of such protection is a reduced access of organic material inside soil voids to decomposing microorganisms. The differences that we are observing in the porosity patterns between $\mathrm{C}$ and $\mathrm{T}$ samples hint at their potentially different effectiveness for protecting carbon. Clearly, T samples with their network of bigger voids will be offering greater microbial access, thus poorer protection than the $\mathrm{C}$ samples that have more porosity connected with smaller features. Observations of Ananyeva et al. (2013) support this hypothesis.

\section{Conclusions}

In this work, we have introduced the essential tools of mathematical morphology in order to quantify the geometrical morphology of soil structure. We made use of 3D images from X-ray CT of soil columns collected at the experimental farm "Finca La Grajera", property of the La Rioja region government, northern Spain. In this study, we considered four columns collected between rows of the vineyard that was established in 1996 with Vitis vinifera L. "Tempranillo". Two types of soil management in between rows were undertaken: $(\mathrm{T})$ conventional tillage management between rows, which consists of a soil tillage of $15-\mathrm{cm}$ depth by cultivator once every 4-6 weeks, as required for weed control during the grapevine growth cycle; (C) permanent cover crop of resident vegetation, which was dominated by annual grass and forbs common to La Rioja.

We have presented the building blocks of mathematical morphology, the morphological operations of dilation, erosion. We have dealt with the Minkowski functionals (i.e. volume, boundary surface, curvature, and connectivity) and the Minkowski functions that take account of the evolution of the Minkowski functionals as morphological operations are performed on the $3 \mathrm{D}$ object of interest with balls of increasing diameter.

Our results suggest that the evolution of morphological features with dilation/erosion is a suitable indicator of soil structure for cultivated soil and it seems to describe the influence of two different soil management practices (i.e. conventional tillage and natural cover crop) on soil structure in a Spanish Mediterranean vineyard. It is worth noting here how these results reflect the different pore structures as
664

\begin{tabular}{|l|lll|}
\hline & Journal : Small 24 & Dispatch : 4-9-2014 & Pages : 15 \\
& Article No. : 928 & $\square$ LE & $\square$ TYPESET \\
& MS Code : PAAG-D-14-00114 & $\downarrow \mathrm{CP}$ & $\checkmark$ DISK \\
\hline
\end{tabular}


depicted by Fig. 5. The homogeneity of the pore space produced by tillage is obvious as compared to the heterogeneity of samples under resident vegetation crop. Similar geometrical features seem to dominate samples $\mathrm{T} 2$ and $\mathrm{C} 2$, but big structures discriminate between them and explain the behavior of specific image porosity and boundary surface when sample T2 is eroded.

These geometrical descriptors that seem to discriminate between these two types of samples could be used as inputs for morphological models of natural soil structures. But further investigations are needed to establish quantitatively the statistical significance of the observed impact of contrasting management practices on soil structure.

\section{Acknowledgments}

This work was partially supported by Plan Nacional de Investigación Científica, Desarrollo e Investigación Tecnológica (I+ D+I) under ref. AGL2011/ 251675 and DGUI (Comunidad de Madrid) and UPM under ref. QM100245066. We thank the staff of the Servicio de Investigación y Desarrollo Tecnológico Agroalimentario (Gobierno La Rioja) for providing the experimental plots and helping with the field work.

Let us be more precise and specify the objects of interest and the geometrical conditions of Hadwiger's theorem. A class of objects to which this theorem applies is the class of sets that can be viewed as the union of a finite number of convex objects. An object $K$ is convex when it contains any point of the segment that joins two of its points. The class of objects made up of finite unions of convex sets is worth considering as any three-dimensional binary image can be considered an element of this class. Binary images are sets of voxels which may be thought of as being cubes, and then any geometrical structure of interest in a binary image is a finite union of convex objects, which are the voxels.
There are three geometrical conditions that a functional to which Hadwiger's theorem applies must fulfill. The first one is motion invariance: the number assigned by a functional must be independent of the position of the object in space when the object is translated or rotated. The second one is $C$-additivity:

$$
\mathcal{F}\left(K_{1} \cup K_{2}\right)=\mathcal{F}\left(K_{1}\right)+\mathcal{F}\left(K_{2}\right)-\mathcal{F}\left(K_{1} \cap K_{2}\right)
$$

That is to say, the number assigned by a functional $\mathcal{F}$ to the union of two objects $K_{1}$ and $K_{2}$ equals the value of the functionals over those two objects minus parts counted twice. And the third condition is continuity. Consider a sequence of objects $\left\{K_{n}\right\}$ that approaches the object $K$ as $n$ tends to infinity. An example of this is the sequence of $r$-parallel bodies of an object $K$; it is clear that the sequence of $r$-parallel bodies $\left\{K_{n}\right\}$ with $r=1 / n$, approaches $K$ as $n$ goes to infinity or, equivalently, as $r$ goes to zero. Then, the continuity condition is fulfilled if $\mathcal{F}\left(K_{n}\right)$ tends to $\mathcal{F}(K)$ as $n$ goes to infinity. Under these conditions there are $d+1$ numbers $c_{i}$ such that

$$
\mathcal{F}(K)=\sum_{i=0}^{d} c_{i} W_{i}^{(d)}(K)
$$

where $W_{i}^{(d)}(K)$ are the Minkowski functionals that assign to any object a number and $K$ belongs to the $d$ dimensional linear space.

\section{Appendix 2}

When the boundary surface of a three-dimensional object is smooth, the third functional, the surface integral of the mean curvature, $M(K)$, may be interpreted as the mean breadth of the object (OSHER and MücKLICH, 2000). This functional might also be an indicator of the surface boundary shape. Points on the boundary surface of an object with positive curvatures settle on convex parts (protrusions) while points with negative curvatures belong to concave parts (hollows). Hence, the mean curvature of convex points will be positive while it will be negative for concave points. Taking into account that the surface integral of the mean curvature over a certain boundary region of $K$ may be interpreted as the average of the mean curvature over this surface region, the third functional, $M(K)$, should

\begin{tabular}{|l|lll|}
\hline & Journal : Small 24 & Dispatch : 4-9-2014 & Pages : 15 \\
& Article No. : 928 & $\square$ LE & $\square$ TYPESET \\
& MS Code : PAAG-D-14-00114 & $\downarrow \mathrm{CP}$ & $\checkmark$ DISK \\
\hline
\end{tabular}


be positive for convex parts of the boundary surface while it should be negative for concave parts.

When the object of interest $K$ corresponds to the pore space $P$, the Euler-Poincaré characteristic $\chi(P)$ is an index of the topology of the pore phase and it quantifies pore connectivity (Vogel and KRETZSCHMAR, 1996). In the plane, Euler-Poincaré can be computed subtracting the number of holes of the object, $H(K)$, from the number of connected components, CC $(K)$ (MECKE, 1998):

$$
\chi(K)=\mathrm{CC}(K)-H(K)
$$

In this context, a connected component of an object is any part of it whose points are connected to one another by curves of points contained in the object. Then, a disk has Euler-Poincaré characteristic equal to 1 because it has one connected component and no holes. A punctured disk has Euler-Poincaré number equal to 0 , a disk punctured twice, -1 , and so on. If the object is just the union of $n$ separated grains on an image, the EulerPoincaré characteristic equals $n$. This object has $n$ connected components. Similar definitions and relations hold in space though distinction between two kinds of holes must be made. In space, the EulerPoincaré characteristic can be computed as the sum of the number of connected components, $\mathrm{CC}(K)$, and the number of cavities of the object, $C(K)$, subtracted by the number of tunnels, $T(K)$ (MECKE, 1998):

$$
\chi(K)=\mathrm{CC}(K)-T(K)+C(K)
$$

Cavities are holes completely surrounded by the object, while tunnels are handles or redundant loops as torus-like holes through the object connected with the exterior or background. If the object is just a separate union of $n$ grains of an image, the Euler-Poincaré characteristic equals $n$. Then, a solid ball has EulerPoincaré characteristic equal to 1 , a ball with a cavity in it, 2, a ball with two cavities, 3, and so on. But, if the ball has a tunnel that goes through it, the EulerPoincaré characteristic is 0 , two tunnels gives a Euler-Poincaré characteristic equal to -1 , and so on.

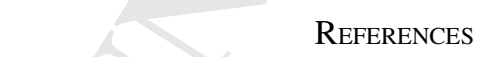

Ananyeva, K., W. Wang, A.J.M. Smucker, M.L. Rivers, A.N. KRAVCHENKO. 2013. Intra-aggregate pore structures are related to total C distribution within soil macro-aggregates. Soil Biology and Biochemistry 57:868-875.

Arns, C.H., M.A. Knackstedt, and K.R. Mecke. 2002. Characterizing the morphology of disordered materials. In: K.R. MECKE and D. Stoyan (Eds.). Morphology of condensed matter. LNP 600. Springer, Berlin. pp. 37-74.

Arns, C.H., M.A. Knackstedt, and K.R. MecKe. 2004. Characterization of irregular spatial structures by parallel sets and integral geometric measures. Colloids and Surfaces A: Physicochem. Eng. Aspects, 241:351-372.

Bossuyt, H., Six, J., Hendrix, P. F., 2002. Aggregate-protected carbon in no-tillage and conventional tillage agroecosystems using carbon-14 labeled plant residue. Soil Science Society America Journal 66, 1965-1973.

BREWER, R. 1964. Fabric and mineral analysis of soils. Wiley, New York.

Chenu, C., Plante, A.F., 2006. Clay-sized organo-mineral complexes in a cultivation chronosequence: revisiting the concept of the primary organo-mineral complex. European Journal of Soil Science 57, 596-607.

Denef, K., Six, J., Bossuyt, H., Frey, S.D., Elliott, E.T., Merckx, R., Paustian, K., 2001. Influence of dry-wet cycles on the interrelationship between aggregate, particulate organic matter, and microbial community dynamics. Soil Biology and Biochemistry 33, 1599-1611.

Ellerbrock, R.H., Gerke, H.H., 2004. Characterizing organicmatter of soil aggregate coatings and biopores by Fourier transform infrared spectroscopy. European Journal of Soil Science 55, 219-228.

Iassonov, P., T. Gebregenus, and M. Tuller. 2009. Segmentation of X-Ray CT Images of Porous Materials: A Crucial Step for Characterization and Quantitative Analysis of Pore Structures. Water Resour. Res. 45: W09415, doi:10.1029/2009WR008087.

Jasinska, E., Baumgartl, T., Wetzel, H., HoRn, R., 2006. Heterogeneity of physico-chemical properties in structured soils and its consequences. Pedosphere 16, 284-296.

Klette, R. And Rosenfeld, A., 2004. Digital geometry. Geometric methods for digital picture analysis. Morgan Kaufmann Series in Computer Graphics and Geometric Modeling, Morgan Kaufmann, San Francisco.

Kravchenko, A.N., Wang, W., Smucker, A.J.M., Rivers, M.L., 2011. Long-term Differences in Tillage and Land Use Affect Intra-aggregate Pore Heterogeneity. Soil Science Society America Journal 75, 1658-1666.

Likos, C. N., Mecke, K. R. and Wagner, H., 1995. Statistical morphology of random interfaces in microemulsions. J. Chem. Phys., 102:9350-9360.

Lehmann, P. 2005. Pore structures: measurement, characterization and relevance for flow and transport in soils. Proc Appl Math Mech 5, 39-42.

Lehmann, P., P. Wyss, A. Flisch, E. Lehmann, P. Vontobel, M. Krafczyk, A. Kaestner, F. Beckmann, A. Gygi, and H. Flühler. 2006. Tomographical imaging and mathematical description of porous media used for the prediction of fluid distribution. Vadose Zone J. 5:80-97.

Matheron, G., 1975. Random sets and integral geometry. Wiley, New York

MECKE, K.R. 1996. Morphological characterization of patterns in reaction-diffusion systems. Phys. Rev. E. 53(5): 4794-4800.

MECKE, K. R. 1998. Integral geometry and statistical physics. Inter. J. Mod. Phys. B. 12(9):861-899.

\begin{tabular}{|l|lll|}
\hline & Journal : Small 24 & Dispatch : 4-9-2014 & Pages : 15 \\
& Article No. : 928 & $\square$ LE & $\square$ TYPESET \\
& MS Code : PAAG-D-14-00114 & $\downarrow \mathrm{CP}$ & $\checkmark$ DISK \\
\hline
\end{tabular}


884

885

886

887

888

889

890

891

892

893

894

895

896

897

898

899

900

901

902

903

904

905

906

907

908

909

910

911

912

913

914

915

916

917

918

919

920

921

922

923

924

925

926

927

928

929

975

976

977
SERRA, J. 1982. Image analysis and mathematical morphology. Academic Press Inc. Orlando, Florida

SeXstone, A.J., Revsbech, N. P., Parkin, T.B., Tiedje J.M., 1985. Direct measurement of oxygen profiles and denitrification rates in soil aggregates. Soil Science Society America Journal 49, 645-651.

Six, J., Elliott, E.T., Paustian, K., 2000. Soil macroaggregate turnover and microaggregate formation: a mechanism for $C$ sequestration under no-tillage agriculture. Soil Biology and Biochemistry 32, 2099-2103.

Sonka, M., V. Hlavac, and R. Boyle. 1998. Image processing, analysis, and machine vision. (2nd ed.) PWS, an Imprint of Brooks and Cole Publishing Inc.

SoILle, P. 2002. Morphological textural analysis: an introduction. In K.R. Mecke and D. Stoyan (eds.). Morphology of condensed matter. LNP 600, pp. 215-237. Springer, Berlin.

Urbanek E., Hallett, P., Feeney D., Horn, R., 2007. Water repellency and distribution of hydrophilic and hydrophobic compounds in soil aggregates from different tillage systems. Geoderma 140, 147-155.

Vogel, H.J., 2002. Topological characterization of porous media. In K.R. MecKE and D. Stoyan (eds.). Morphology of condensed matter. LNP 600, pp. 75-92. Springer, Gerlin

Vogel, H.J., H. Hoffmann, and K. Roth. 2005. Studies of crack dynamics in clay soil. I. Experimental methods, results, and morphological quantification. Geoderma 125:203-211.

Vogel, J.H., U. Weller, and S. Schlüter. 2010. Quantification of soil structure based on Minkowski functions. Comput. Geosci. 36:1236-1245.

von LÜtZow, M., KöGel-Knabner, I., Ekschmitt, K., Matzner, E., Guggenberger, G., Marschner, B., Flessa, H., 2006. Stabilization of organic matter in temperate soils: mechanisms and their relevance under different soil conditions-a review. European Journal of Soil Science 57, 426-445.

W ANG, M., and Сн.H LAI. 2009. A Concise Introduction to Image Processing using $\mathrm{C}++$ Chapman \& Hall/CRC (Numerical Analysis and Scientific Computing Series).

Wang, W., Kravchenko, A.N., Smucker, A.J.M., Liang, W., RivERS, M.L., 2012. Intra-aggregate pore characteristics: X-ray computed microtomography analysis. Soil Science Society America Journal 76, 1159-1171.

Zhou, H, X. Peng, E. Perfect, T. Xiao, and G. Peng. 2013. Effects of organic and inorganic fertilization on soil aggregation in an Ultisol as characterized by synchrotron based X-ray microcomputed tomography. Geoderma 195-196: 23-30.
930

931

932

933

934

935

936

937

938

939

940

941

942

943

944

945

946

947

948

949

950

951

952

953

954

955

956

957

958

959

960

961

962

963

964

965

966

967

968

969

970

971

972

973

974
Santos, D., Murphy, S.L.S., Taubner, H., Smucker, A.J.M., Horn R., 1997. Uniform separation of concentric surface layers from aggregates. Soil Science Society America Journal 61, 720-724.

\begin{tabular}{|l|lll|}
\hline & Journal : Small 24 & Dispatch : 4-9-2014 & Pages : 15 \\
Article No. : 928 & $\square$ LE & $\square$ TYPESET \\
& MS Code : PAAG-D-14-00114 & $\checkmark \mathrm{CP}$ & $\downarrow$ DISK \\
\hline
\end{tabular}

